\title{
ADAPTIVE ELECTRONIC PROCESSING IN OPTICAL PMD-IMPAIRED SYSTEMS
}

\author{
T. Foggi ${ }^{1}$, G. Colavolpe ${ }^{2}$, E. Forestieri ${ }^{1,3}$, and G. Prati ${ }^{1,3}$ \\ ${ }^{1}$ CNIT, Photonic Networks National Lab, Pisa, Italy \\ ${ }^{2}$ Università di Parma, Dipartimento di Ingegneria dell'Informazione, Parma, Italy \\ ${ }^{3}$ Scuola Superiore Sant'Anna, Pisa, Italy \\ tommaso.foggi@cnit.it
}

\begin{abstract}
In high-speed optical transmission systems, one of the most challenging impairments is represented by the signal distortions produced by polarization-mode dispersion (PMD). In this paper, we analyze the limits of electronic compensation schemes, in the form of maximum-likelihood sequence detection receivers. With a constraint on the front-end processing after photo-detection, we compute the signal statistics necessary to implement this strategy. The receiver adaptivity is also discussed. The relevant performance is analyzed by means of computer simulations and accurate analytical performance bounds, and compared with the performance of all-optical compensators and decision-feedback equalizers. A significant performance loss with respect to optical compensation is observed for large values of the differential group delay (DGD) showing that, after the non reversible transformation operated by the photo-detector, it is not possible to effectively cope with the PMD-induced impairments.
\end{abstract}

\section{INTRODUCTION}

In recent years, the application of electronic processing to optical preamplified transmission systems has been deeply investigated in order to combat linear and non-linear impairments of the optical channel. In fact, although optical compensation has demonstrated to be very efficient in recovering signals affected by heavy penalties induced by PMD (see [1] and references therein), these devices need advanced and cost-ineffective technologies [2], so that the use of well-known and popular electronic schemes is of great interest. In the first-order approximation, the PMD effects are represented by the introduction of a differential group delay (DGD) between the two principal states of polarization (PSPs), causing intersymbol interference (ISI). Classical methods of 
electronic processing of ISI-affected signals have been applied, such as linear equalization [3], and non-linear cancellation has also been used since the optical to electrical conversion causes a non-linear transformation of the signal [4]. Recent studies have focused on the comparison between these equalization techniques and optical compensation [5], evidencing qualities and lacks of both solutions.

More effective electronic techniques, based on maximum-likelihood sequence detection (MLSD) implemented through the Viterbi algorithm (VA) [6], have been proposed in [2,3,5]. In [3], the presence of optical amplifiers is not considered. As a consequence, the amplified spontaneous emission (ASE) noise is not present and the statistics of the received signal, necessary to compute the VA branch metrics are conditionally Gaussian due to shot and thermal noise. Since making a Gaussian assumption for noise statistics after photo-detection when ASE noise is dominant leads to inaccurate results in the computation of the receiver metrics [7], in [2,5] the statistics of the received signal are approximately measured and updated in real-time during transmission and assuming no decision errors. This method has been compared with classical equalization schemes in $[2,5]$, showing that a better performance, as expected, can be obtained by using the MLSD strategy.

In this paper, we derive a practically optimal receiver, in the sense of MLSD, with the constraint of one sample per bit at the output of the postdetection filter. The exact statistics of the received signal are computed and the branch metrics of the VA expressed accordingly. A closed-form approximation of these branch metrics, which entails a negligible performance loss, is also given. Based on the exact branch metric computation, analytical bounds for the system performance are also provided, allowing to reach values of bit error rate (BER) below $10^{-12}$. This analytical method also represents an interesting tool for optimizing the receiver parameters without resorting to time-consuming computer simulations. Since PMD is a time-varying phenomenon, the receiver has also to adaptively update some parameters. This aspect is also discussed. A comparison with commonly adopted electronic equalization and optical compensation techniques is also provided showing that after the irreversible transformation given by the photodetector, the receiver is not able to effectively cope with the PMD distortions.

\section{SYSTEM MODEL}

The considered transmission system is shown in Fig. 1, where double arrows denote two orthogonal states of polarization (SOPs) in the fiber. The optical signal generated by a laser modulator is launched in a single-mode fiber (SMF) operating in linear regime. At the receiver end, after a flat-gain optical amplifier (OA), which also introduces ASE noise, the received signal is opti- 


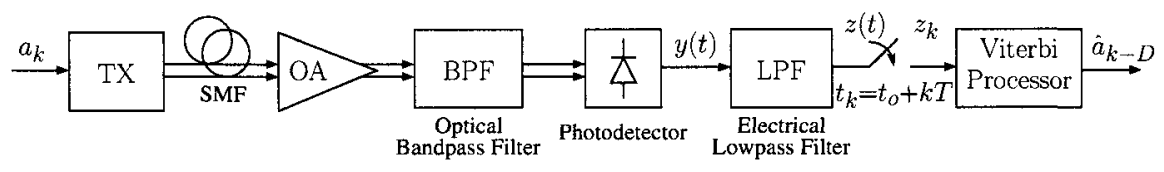

Figure 1. System model.

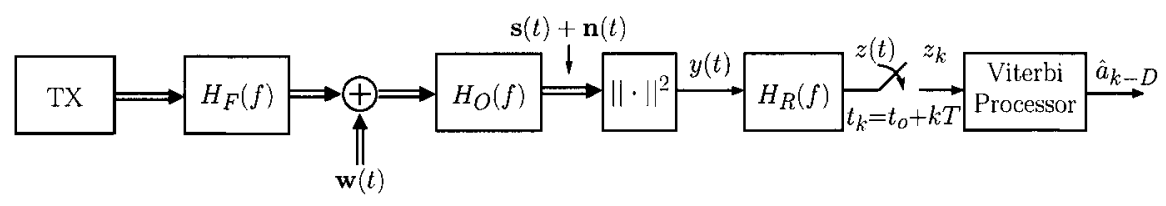

Figure 2. Low-pass equivalent of the considered system.

cally filtered prior photo-detection. The photo-detection process and the following electronic circuitry will add shot and thermal noise, respectively. The ASE noise is modeled as additive white Gaussian noise (AWGN). By using high-gain amplifiers we can assume that the other sources of noise are negligible. The detected signal $y(t)$ is then low-pass filtered and the resulting signal $z(t)$ is sampled at one sample per bit interval $T$. Finally, a Viterbi processor elaborates the received sequence $\mathbf{z}=\left\{z_{k}\right\}$ in order to take reliable decisions on the transmitted bit sequence $\mathbf{a}=\left\{a_{k}\right\}$.

The low-pass equivalent model of the system is shown in Fig. 2. At the optical filter output, the components of the two-dimensional complex vectors $\mathbf{s}(t)=\left(s_{1}(t), s_{2}(t)\right)$ and $\mathbf{n}(t)=\left(n_{1}(t), n_{2}(t)\right)$ represent the useful signal and noise components on two orthogonal SOPs, respectively. The noise components are Gaussian but not white, since they are obtained by filtering the low-pass equivalent AWGN $\mathbf{w}(t)=\left(w_{1}(t), w_{2}(t)\right)$. In the following, we will assume that $w_{1}(t)$ and $w_{2}(t)$ have two-sided power spectral density $N_{0}$. The low-pass equivalent frequency response of fiber, optical and postdetection filters are denoted by $H_{F}(f), H_{O}(f)$, and $H_{R}(f)$, respectively. At the output of the photodiode the detected signal can be described as the sum of two contributions, one for each SOP:

$$
y(t)=\|\mathbf{s}(t)+\mathbf{n}(t)\|^{2}=\left|s_{1}(t)+n_{1}(t)\right|^{2}+\left|s_{2}(t)+n_{2}(t)\right|^{2} .
$$

Clearly, after photo-detection the noise becomes signal-dependent and its statistics change. In the following, optical and postdetection filter parameters can be chosen arbitrarily, since the proposed receiver is independent of a particular choice of the filter shape or bandwidth. 


\section{MLSD RECEIVER}

With the constraint on the above receiver structure and in particular on the use of one sample per bit interval at the output of the postdetection filter, the optimal MLSD receiver is based on the following strategy [6]

$$
\hat{\mathbf{a}}=\arg \max _{\mathbf{a}} p(\mathbf{z} \mid \mathbf{a}) \text {. }
$$

By means of computer simulations, we verified that for the practically used optical and postdetection filters, conditioning on the transmitted sequence the samples $z_{k}$ can be considered independent. Hence, the conditional probability density function (pdf) $p(\mathbf{z} \mid \mathbf{a})$ given the transmitted sequence can be expressed as

$$
p(\mathbf{z} \mid \mathbf{a})=\prod_{k} p\left(z_{k} \mid \mathbf{a}\right) .
$$

Assuming that the system is causal and with finite memory $L$, it will be $p\left(z_{k} \mid \mathbf{a}\right)=$ $p\left(z_{k} \mid a_{k}, a_{k-1}, \ldots, a_{k-L}\right)$. Therefore, the optimal MLSD algorithm can be efficiently implemented by means of the VA with the following branch metrics:

$$
\lambda_{k}\left(a_{k}, \sigma_{k}\right)=\ln p\left(z_{k} \mid a_{k}, \sigma_{k}\right)
$$

having defined the trellis state as $\sigma_{k}=\left(a_{k-1}, a_{k-2}, \ldots, a_{k-L}\right)$. Hence, the number of states $S=2^{L}$ increases exponentially with the channel memory $L$.

A closed-form expression for the $2 S$ pdfs in (4), necessary to implement the optimal MLSD strategy, does not exist. ${ }^{1}$ We have numerically evaluated all these necessary conditional pdfs by using the method described in [8] and stored them in a look-up table that will be addressed, in order to compute branch metrics, by the received samples properly quantized and by the considered trellis transition. Although from an implementation point of view this solution is not feasible, since entire pdfs must be stored, we have followed this approach in order to investigate the ultimate performance of the MLSD strategy. The obtained results also represent a benchmark for alternative solutions based on an approximate, possibly closed-form, branch metrics expression. In particular, we use the following non-central chi-square approximation $[7,9]$

$$
\begin{aligned}
p\left(z_{k} \mid a_{k}, \sigma_{k}\right) \simeq & \frac{1}{N_{0}}\left(\frac{z_{k}}{s_{R}\left(a_{k}, \sigma_{k}\right)}\right)^{\frac{\nu-1}{2}} \exp \left(-\frac{z_{k}+s_{R}\left(a_{k}, \sigma_{k}\right)}{N_{0}}\right) \\
& \cdot I_{\nu-1}\left(\sqrt{\frac{2 z_{k} s_{R}\left(a_{k}, \sigma_{k}\right)}{N_{0}}}\right)
\end{aligned}
$$

\footnotetext{
${ }^{1}$ It is well known that conditioning on the transmitted sequence, the samples at the photo-detector output have non-central chi-square distribution [6]. However, the presence of the electrical filter modifies these statistics.
} 
in which $I_{\nu-1}(x)$ is the modified Bessel function of first kind and order $\nu-1$, $\nu=\frac{B_{O}}{B_{R}}$, where $B_{O}$ and $B_{R}$ are the optical and postdetection filter noise equivalent bandwidths, respectively, and $s_{R}\left(a_{k}, \sigma_{k}\right)$ is the output of filter $H_{R}(f)$, sampled at time $t_{k}$, in the absence of noise. This term obviously depends not only on the considered optical and postdetection filters but also on the PMD parameters. Although we verified that (5) is not suitable for BER evaluation in standard receivers with arbitrary filters (the BER would be heavily underestimated), its use to approximate the branch metrics expression produces a negligible performance loss with respect to the optimal solution, as it will be shown in the numerical results.

As already mentioned, the number of trellis states, and thus the complexity, depends exponentially on the channel memory $L$. For commonly used optical and electrical filters, and DGD values lower than a bit interval, we verified that $L \leq 3$. Hence the number of states is at most $S=8$. In addition, the application of reduced-state sequence detection (RSSD) techniques [10] allows to substantially reduce the number of trellis states. In particular, a reduced state $\sigma_{k}^{\prime}=\left(a_{k-1}, a_{k-2}, \ldots, a_{k-L^{\prime}}\right)$, with $L^{\prime}<L$, may be defined. The resulting number of states is reduced to $2^{L^{\prime}}<2^{L}$. In order to compute the branch metrics (4) in a reduced trellis, the necessary symbols not included in the state definition may be found in the survivor history [10]. We note that, in the limiting case of $L^{\prime}=0$, the trellis diagram degenerates and symbol-by-symbol detection with decision feedback is performed. The resulting receiver can be considered as a non linear equalizer with decision feedback. Our numerical results, not shown here for a lack of space, show that the resulting performance loss is negligible.

Since PMD is a time-varying phenomenon, the receiver parameters should be adaptively updated. By using the above mentioned approximated closedform expression of the branch metrics, when PMD changes the receiver has to simply adaptively identify the term $s_{R}\left(a_{k}, \sigma_{k}\right)$. This can be easily done by using a gradient adaptation algorithm and, as a cost function, the one defining the non linear branch metrics.

\section{PERFORMANCE ANALYSIS}

The classical union bound on the bit error probability $P_{b}$ has expression [6]

$$
P_{b} \leq \sum_{\mathbf{a}} P(\mathbf{a}) \sum_{\hat{\mathbf{a}} \neq \mathbf{a}} b(\mathbf{a}, \hat{\mathbf{a}}) P(\mathbf{a} \rightarrow \hat{\mathbf{a}})
$$

in which $\mathbf{a}=\left\{a_{k}\right\}$ and $\hat{\mathbf{a}}=\left\{\hat{a}_{k}\right\}$ denote the bit sequences corresponding to the correct and erroneous paths, respectively, $b(\mathbf{a}, \hat{\mathbf{a}})$ is the number of bit errors entailed by the error event $(\mathbf{a}, \hat{\mathbf{a}}), P(\mathbf{a} \rightarrow \hat{\mathbf{a}})$ is the pairwise error probability and $P(\mathbf{a})$ is the a priori probability of sequence $\mathbf{a}$. The pairwise error probabil- 
ity $P(\mathbf{a} \rightarrow \hat{\mathbf{a}})$ is the probability that the sum of the branch metrics relative to the erroneous path exceeds the sum of the branch metrics on the correct path. This probability may be easily computed by employing the same method described in [8] already used to compute the exact expression of the conditional density functions $p\left(z_{k} \mid \mathbf{a}\right)$.

From the union bound, we derive a lower and an approximated upper bound for the bit error probability. The lower bound is simply obtained by considering the most likely error event. The approximated upper bound is obtained by truncating the union bound considering a few most frequently occurring error events only. The accuracy of these bounds will be shown in the numerical results.

\section{NUMERICAL RESULTS}

In this section, the performance of the considered detection schemes is assessed in terms of BER versus $E_{b} / N_{0},{ }^{2} E_{b}$ being the received signal energy per information bit. In all simulations, the optical filter is assumed to be a fourth order Butteworth filter with low-pass equivalent bandwidth equal to $0.95 / T$, whereas the postdetection filter is a fifth order Bessel filter with bandwidth $0.75 / T$.

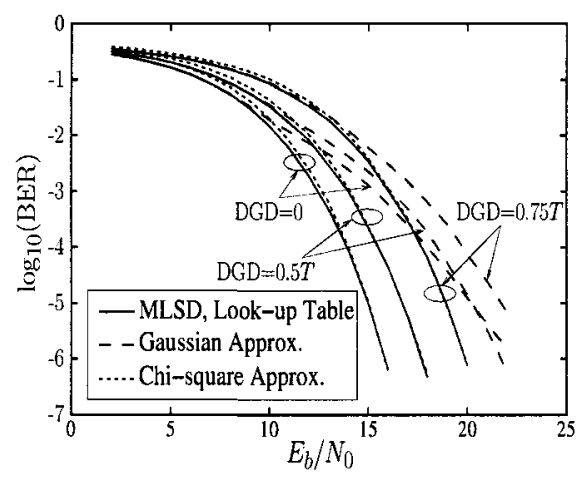

Figure 3. Performance of the VA with exact, chi-square, and Gaussian metrics.

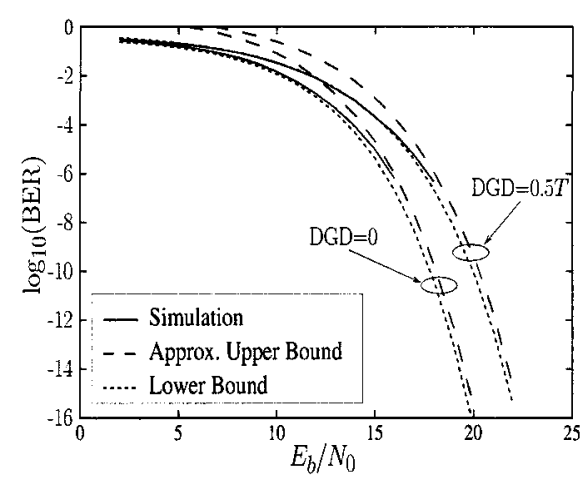

Figure 4. Lower bound and and approximated upper bound.

In Fig. 3, we show the performance of the VA when the exact and approximated branch metrics are used. First-order PMD, with power splitting equal to 0.5 and different values of the DGD, is considered. It can be observed that the chi-square closed-form approximation (5) entails a negligible performance

\footnotetext{
${ }^{2}$ The ratio $E_{b} / N_{0}$ is proportional to the optical signal-to-noise ratio and represents the number of detected photons per bit at the input of the OA.
} 
loss with respect to the optimal solution, especially for low BER values. On the contrary, when a Gaussian approximation for the branch metrics is used [3], the performance loss is remarkable (see Fig. 3).

The accuracy of the described lower and approximated upper bounds is shown in Fig. 4. In this case also, first-order PMD is considered with DGD values of 0 and $0.5 T$. It is clear that it is not necessary to have recourse to timeconsuming computer simulations since this tool predicts very well the receiver performance and can be also used to optimize the system parameters.

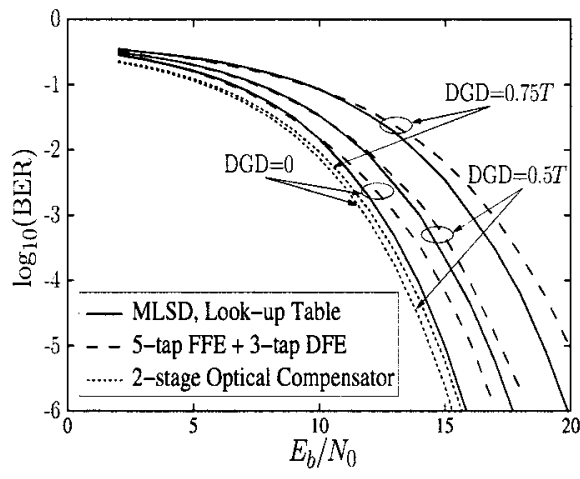

Figure 5. Comparison between optical and electronic compensation structures.

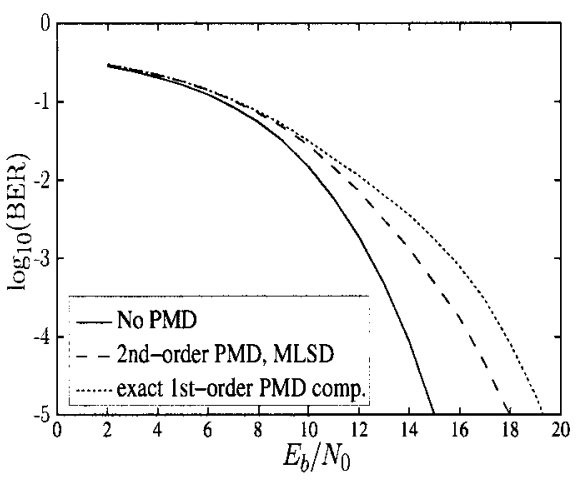

Figure 6. Performance in the case of second-order only PMD.

In Fig. 5, we compare the performance of the MLSD approach with that of optical and electronic compensators. The considered optical compensator consists of a cascade of some optical devices. The first optical device is a polarization controller (PC) which allows us to modify the polarization of the optical signal at its input. Then we have in this example two polarization maintaining fibers (PMF) separated by another PC. A PMF introduces a DGD between the components of the optical signal on the two orthogonal SOPs corresponding to its slow and fast axes. In the considered example, the DGD of the considered PMFs is $0.5 T$. The other considered electronic compensation scheme is a $T / 2$-spaced decision-feedback equalizer (DFE) with a feedforward filter with 5 taps and a feedback filter with 3 taps. No performance improvement has been observed by considering a more complex equalizer. In this case also, the presence of first-order PMD is considered. As it can be observed, the optical compensator is able to cope with the PMD effects almost perfectly. On the contrary, although MLSD performs better than equalization, electronic processing schemes, operating after the non-reversible transformation by the photodiode, entail a ramarkable penalty.

Finally, in Fig. 6 we assume that the first-order effects have already been perfectly compensated and that the residual second-order parameters are the 
following: DGD derivative equal to $0.2 T^{2}$ and PSP rotation rate equal to $0.4 T$ (equal signal power splitting among the PSPs is also considered). The MLSD receiver is able to recover only 1 of the $4 \mathrm{~dB}$ penalty produced by the $2 \mathrm{nd}$ order PMD. Although we point out that only the outage probability can give a final answer, it seems that electronic compensation cannot be considered a viable solution not even joint with a simple optical scheme which perfectly compensates the effects of the first order PMD.

\section{CONCLUSIONS}

In this paper, the limits of electronic compensation have been shown. With a constraint on the receiver structure, the optimal MLSD receiver has been derived. A practically optimal closed-form branch metrics expression has been also given and accurate performance bounds computed. Comparisons with other equalization and optical compensation schemes have been performed showing that optical compensation is the only viable solution to effectively cope with the PMD effects.

\section{REFERENCES}

[1] E. Forestieri, G. Colavolpe, and G. Prati, "Novel MSE adaptive control of optical PMD compensator," J. Lightwave Technol., vol. 20, pp. 1997-2003, Dec. 2002.

[2] H. F. Haunstein, W. Sauer-Greff, A. Dittrich, K. Sticht, and R. Urbansky, "Principles for electronic equalizationof polarization-mode dispersion," J. Lightwave Technol., vol. 22, pp. 1169-1182, Apr. 2004.

[3] J. H. Winters and R. D. Gitlin, "Electrical signal processing techniques in long-haul fiberoptic systems," IEEE Trans. on Commun., vol. 38, pp. 1439-1453, Sept. 1990.

[4] J. H. Winters and S. Kasturia, "Adaptive nonlinear cancellation for high-speed fiber-optic systems," J. Lightwave Technol., vol. 10, pp. 971-977, July 1992.

[5] F. Buchali and H. Bulow, "Adaptive PMD compensation by electrical and optical techniques," J. Lightwave Technol., vol. 22, pp. 1116-1126, Apr. 2004.

[6] J. Proakis, Digital Communication. New York: McGraw-Hill, 3rd ed., 1996.

[7] Y. Cai, J. M. Morris, T. Adali, and C. R. Menyuk, "On turbo code decoder performance in optical-fiber communication systems with dominating ASE noise," J. Lightwave Technol., vol. 21, pp. 727-734, Mar. 2003.

[8] E. Forestieri and G. Prati, "Exact analytical evaluation of second-order PMD impact on the outage probability for a compensated system," J. Lightwave Technol., vol. 22, pp. 988-996, Apr. 2004.

[9] P. A. Humblet and M. Azizoglu, "On the bit error rate of lightwave systems with optical amplifiers," J. Lightwave Technol., vol. 9, pp. 1576-1582, Nov. 1991.

[10] M. V. Eyuboğlu and S. U. Qureshi, "Reduced-state sequence estimation with set partitioning and decision feedback," IEEE Trans. on Commun., vol. 38, pp. 13-20, Jan. 1988. 